BMJ Open Diabetes Research \& Care

\title{
Sitagliptin versus mitiglinide switched from mealtime dosing of a rapid-acting insulin analog in patients with type 2 diabetes: a randomized, parallel-group study
}

\author{
Yumie Takeshita, ${ }^{1}$ Toshinari Takamura, ${ }^{2}$ Yuki Kita, ${ }^{1}$ Akiko Takazakura, ${ }^{1}$ \\ Ken-ichiro Kato, ${ }^{1}$ Yuki Isobe, ${ }^{1}$ Shuichi Kaneko ${ }^{1}$
}

To cite: Takeshita $Y$, Takamura T, Kita Y, et al. Sitagliptin versus mitiglinide switched from mealtime dosing of a rapid-acting insulin analog in patients with type 2 diabetes: a randomized, parallel-group study. BMJ Open Diabetes Research and Care 2015;3: e000122. doi:10.1136/ bmjdrc-2015-000122

\section{- Additional material is available. To view please visit the journal (http://dx.doi.org/ 10.1136/bmjdrc-2015- 000122)}

YT and TT contributed equally.

Received 9 June 2015 Revised 14 July 2015 Accepted 20 July 2015

\section{(1) crossarath}

\begin{abstract}
${ }^{1}$ Department of Disease Control and Homeostasis, Kanazawa University Graduate School of Medical Sciences, Kanazawa, Ishikawa, Japan ${ }^{2}$ Department of Comprehensive Metabology, Kanazawa University Graduate School of Medical Sciences, Kanazawa, Ishikawa, Japan
\end{abstract}

\section{ABSTRACT}

Purpose: We determined the feasibility of substituting sitagliptin or mitiglinide for bolus insulin injection therapy in patients with type 2 diabetes.

Methods: 60 patients with type 2 diabetes were enrolled and randomized to switch from mealtime dosing of a rapid-acting insulin analog to either sitagliptin or mitiglinide for 16 weeks.

Results: Body weight, body mass index, and waist circumference decreased significantly in both groups at the end of the study. Mitiglinide significantly increased fasting plasma glucose (FPG) levels at the end of the study from $146.5 \pm 36.3$ to 168.0 $\pm 38.8 \mathrm{mg} / \mathrm{dL}$, whereas sitagliptin did not affect FPG. Glycated hemoglobin (HbA1c) and 1,5-anhydroglucitol increased significantly in both groups. The $C$ peptide immunoreactivity (CPR) responses after arginine were diminished in both groups. $\gamma$-GTP and triglycerides increased, and high-density lipoprotein cholesterol and adiponectin decreased, in the sitagliptin group, but not in the mitiglinide group. Mean Diabetes Treatment Satisfaction Questionnaire scores improved significantly in both groups. Patients whose mean total daily doses of rapid-acting insulin analog were 16.6 and 17.8 units were switched to sitagliptin and mitiglinide, respectively, without a change in the HbA1c level. Total insulin doses/body weight predicted changes in $\mathrm{HbA1C}$ only in the sitagliptin group, but not in the mitiglinide group. Use of $>0.27 \mathrm{IU} / \mathrm{kg}$ of a rapid-acting insulin analog predicted an increase in $\mathrm{HbA1C}$ after switching to sitagliptin. The CPR index (CPI) was also a predictor for a change in $\mathrm{HbA1C}$ in the sitagliptin group, but not in the mitiglinide group; patients with a $\mathrm{CPI}<1.4$ developed a worse $\mathrm{HbA1C}$ after switching to sitagliptin.

Conclusions: Sitagliptin may predominantly act on FPG, whereas mitiglinide may act on postprandial plasma glucose to achieve glycemic control after switching from a bolus insulin regimen. Additional therapy to sitagliptin or mitiglinide is clearly required to obtain equivalent glycemic control in patients using a higher dose of insulin.

Trial registration number: (UMIN 000007051)

\section{Key messages}

- This is the first report showing the feasibility of substituting oral hypoglycemic agents for insulin injection therapy in an open-label randomized, parallel-group study. The predictive variable for a change in glycated hemoglobin (HbA1C) was total insulin dose before switching.

- Patients whose mean total daily doses of rapid-acting insulin analog were 16.6 and 17.8 units were switched to sitagliptin and mitiglinide, respectively, without a change in the $\mathrm{HbA1c}$ level. Use of $>0.27 \mathrm{IU} / \mathrm{kg}$ of a rapid-acting insulin analog predicted an increase in $\mathrm{HbA1C}$ after switching to sitagliptin.

- Sitagliptin, but not mitiglinide, may exert unique pleiotropic effects on fatty acid composition. After the switch from insulin, sitagliptin significantly decreased the $\Delta 5$ desaturase, whereas it significantly increased the $\Delta 6$ desaturase.

- Patients with a $\mathrm{C}$ peptide immunoreactivity index (CPI) $<1.4$ had worse HbA1c levels after switching to sitagliptin. Our study is the first to prospectively demonstrate the usefulness of the $\mathrm{CPI}$ for "tailor-made" diabetic medicine.

\section{INTRODUCTION}

As the population with diabetes increases, the number of individuals receiving insulin injections increases proportionally. Rapidacting insulin analogs have been particularly effective at targeting postprandial hyperglycemia as well as nadir fasting plasma glucose (FPG) ${ }^{1-3}$ In contrast, excess insulin/insulinlike growth factor signaling accelerates cellular aging by negatively regulating FOXO transcription factors. ${ }^{4}$ Aging phenotypes include cancer and dementia. The risk for liver cancer increases particularly in patients with diabetes undergoing insulin treatment. ${ }^{5-7}$ Several studies have identified hyperinsulinemia as a risk factor for 
accelerated cognitive decline and dementia. ${ }^{8-10}$ Indeed, the risk of dementia is highest in patients with diabetes treated with insulin. ${ }^{11-13}$ Insulin injection therapy is also associated with pain and places a heavy physical, mental, and financial burden on patients. Similarly, recent large-scale clinical trials have suggested that intensive antidiabetic therapies that cause unnecessary hyperinsulinemia do not result in satisfactory cardiovascular outcomes in patients with type 2 diabetes and may cause hypoglycemia and weight gain. ${ }^{14}$

One possible solution for this paradox came from the launch of incretin-based agents because they avoid unnecessary hyperinsulinemia and thereby avoid hypoglycemia and weight gain. Sitagliptin and mitiglinide are major agents for switching from insulin injections. Sitagliptin increases insulin secretion and decreases glucagon concentration in a glucose-dependent manner. ${ }^{15}$ Its use results in a lower incidence of hypoglycemia compared with that of other oral hypoglycemic agents (OHAs). ${ }^{16}{ }^{17}$ However, mitiglinide provokes rapid and short-acting insulin secretion that improves postprandial hyperglycemia and mimics normal physiological insulin secretion and glucose metabolism in healthy individuals. Owing to its shorter duration of action, mitiglinide has a lower risk of hypoglycemia compared with other insulin secretagogues. ${ }^{18}$

From the perspective of switching from insulin treatment to an OHA, it is prudent to carefully consider the inclusion criteria. This approach may improve blood sugar control in patients with diabetes and help reduce the overall cost of medical care. Additionally, it remains unclear what clinical features of patients, including diabetes duration, dose of insulin, insulin secretory capacity, body composition, and fatty acid composition, are associated with changes in glycated hemoglobin (HbA1c) after switching from a bolus insulin regimen to an OHA. For these reasons, we determined the feasibility of substituting an OHA for insulin injection therapy in patients with type 2 diabetes.

\section{METHODS}

\section{Overview}

This was a randomized, parallel-group study conducted on Japanese patients. This study was designed in accordance with the principles stated in the Declaration of Helsinki, and the protocol was reviewed and approved by the Ethics Committee of Kanazawa University Hospital, Ishikawa, Japan. Patients provided written informed consent before participation.

Sixty patients with type 2 diabetes and on rapid-acting insulin injection therapy were recruited from our Department of Disease Control and Homeostasis from May 2010 to July 2011. This trial is registered with the University Hospital Medical Information Network Clinical Trials Registry, number +000007051 .

\section{Patient eligibility}

Inclusion criteria were: (1) males and females with type 2 diabetes (age, >20 years); (2) rapid-acting insulin dosage $<60$ units/24 h; and (3) moderately controlled diabetes with a change in $\mathrm{HbA} 1 \mathrm{c}<3 \%$ in the 12 weeks before screening. We used this criterion according to the previous clinical trials, in which the inclusion criteria involve a $3-4.5 \%$ range of HbA1c. For example, the LEAD-1 SU study included patients with type 2 diabetes with HbAlc $7.0-11.0 \%$ or $7.0-10.0 \% .^{19}$ Another study included patients who met the following inclusion criteria during the screening period: HbAlc 7-11.5\% at 4 weeks before randomization, HbAlc $7-11 \%$ at 1 week before randomization. ${ }^{20}$ Accordingly, we maximally allowed a $<3 \%$ change in HbA1c for 12 weeks before screening. Exclusion criteria were: (1) hypersensitivity or contraindication to mitiglinide or sitagliptin; (2) a history of type 1 diabetes or a history of ketoacidosis; (3) experienced repeated episodes of unexplained hypoglycemia as defined by an FPG or without symptoms of hypoglycemia or $<60 \mathrm{mg} / \mathrm{dL}$ with symptoms of hypoglycemia; (4) concomitantly suffering from infection or planning to have surgery; (5) treatment with a mitiglinide or sitagliptin within 12 weeks before screening; (6) concomitant corticosteroid therapy; (7) poorly controlled diabetes (with ketoacidosis or with an increase in HbAlc $>3 \%$ in the 12 weeks before screening); (8) undergoing dialysis, and serum creatinine $>2.5 \mathrm{mg} / \mathrm{dL}$ in men or $>2.0 \mathrm{mg} / \mathrm{dL}$ in women; (9) alanine aminotransferase and/or aspartate aminotransferase (AST) levels more than 2.5-fold the upper limit of normal; (10) poorly controlled hypertension, systolic blood pressure $>160 \mathrm{~mm} \mathrm{Hg}$, or diastolic blood pressure >100 mm Hg; (11) currently and/or previously suffering from heart failure; (12) severe retinopathy; (13) malignancy on active therapeutic regimen or without complete remission or cure; (14) pregnancy or breast feeding; and (15) some barrier to participation in the study, as assessed by the investigators.

\section{Participants \\ Efficacy end points}

The primary efficacy end point was the change in FPG from baseline to week 16 . The secondary end point assessed at week 16 was the change in HbAlc from baseline. Other end points included blood 1,5-anhydroglucitol, fasting lipids, and insulin. The $\mathrm{C}$ peptide immunoreactivity (CPR) index (CPI) was calculated using the formula: [100×fasting CPR $(\mathrm{ng} / \mathrm{mL})] /$ $[18 \times$ FPG $(\mathrm{mm})] .{ }^{21} \mathrm{C}$ peptide and insulin levels were determined by an immunoenzymometric assay using Tosoh kits (Shunan, Japan). The lower limit of quantification for CPR was $0.2 \mathrm{ng} / \mathrm{mL}$. The intra-assay and interassay coefficients of variation were $<6 \%$. Glucose and HbA1C were measured by standard methods. The results of a physical examination, vital signs (blood pressure), body weight, and laboratory evaluations, including 
hematology, serum chemistry, and urinalysis, were recorded throughout the study.

Serum fatty acid levels were measured as a secondary outcome. A serum sample (approximately $0.2 \mathrm{~mL}$ ) and $2 \mathrm{~mL}$ chloroform-methanol (2:1) were placed in a Pyrex centrifuge tube, homogenized with a Polytron homogenizer (PCU-2-110; KINEMATICA GmbH, Lucerne, Switzerland), and centrifuged at $3000 \mathrm{rpm}$ for $10 \mathrm{~min}$. An aliquot of the chloroform-methanol extract was transferred to another Pyrex tube and dried under a nitrogen gas stream. The dried sample was dissolved in a $100 \mu \mathrm{L}$ $0.4 \mathrm{M}$ potassium methoxide methanol $/ 14 \%$ boron trifluoride-methanol solution, and the fatty acid concentration was measured at SRL Inc. by gas chromatography (Shimizu GC 17A, Kyoto, Japan). The $\Delta 5$ desaturase (D5D) activity index and the $\Delta 6$ desaturase (D6D) activity index were expressed as the arachidonic acid to dihomo- $\gamma$-linolenic acid ratio and the $\gamma$-linolenic acid ratio to the linoleic acid ratio in blood, respectively.

Safety and tolerability were assessed by reviewing several safety parameters. Key safety parameters included general adverse experiences (AEs), treatment discontinuations, hypoglycemic events, and hyperglycemic events, as well as other parameters of interest, such as body weight and urinary ketones. AEs were monitored throughout the study and were rated by the investigators for intensity and their relationship to the study drug. AEs with an onset date up to 2 weeks after concluding treatment were evaluated in person.

A computer-generated dynamic randomization sequence assigned participants in a 1:1 ratio to treatment in the sitagliptin or mitiglinide group to adjust for demographic differences (age, insulin dose, and HbA1c) between the agents' groups. The study continued in an open-label fashion for an additional 16 weeks as described. Combination therapy was initiated on the day on which insulin injection therapy was completely withdrawn. The concomitantly used OHAs and medications other than hypoglycemic agents were continued after the switch. Patients remained on stable doses of the medications during the study period. The investigators did not use rescue medication such as insulin or sulfonylureas at any time after randomization even if patients did not achieve a satisfactory therapeutic effect.

All patients and responsible guardians underwent $1 \mathrm{~h}$ of nutritional counseling with an experienced dietician before the study. In addition, all patients were given a standard calorie diet $(30 \mathrm{kcal} / \mathrm{kg} /$ day; $50-60 \%$ carbohydrate, $20-30 \%$ fat, and $15-20 \%$ protein) and exercise (5-6 metabolic equivalent estimations for $30 \mathrm{~min}$ daily) counseling before the study.

\section{Arginine-stimulation test}

The arginine-stimulation test has been demonstrated to be a valid method for evaluating residual $\beta$-cell function, even during periods of hyperglycemia. ${ }^{22}$ We previously showed that arginine-evoked insulin secretion predicts the requirement for basal insulin replacement in patients with type 2 diabetes. ${ }^{1}$ In the present study, we tested the hypothesis whether the $\beta$-cell function evaluated with the arginine-stimulation test predicts the effects of sitagliptin or mitiglinide on HbAlc after switched from insulin therapy. Patients were kept still for 30 min after an overnight fast, and CPR were assessed at the preloading baseline $(0 \mathrm{~min})$. Arginine $(30 \mathrm{~g})$ was administered intravenously by infusing a $10 \%$ L-arginine hydrochloride solution over $30 \mathrm{~min}$. Blood was collected at seven time points: preloading $(0 \mathrm{~min})$ and $15,30,45$, 60,90 , and $120 \mathrm{~min}$ after arginine loading. Circulating CPR was measured at each time point and used to construct an arginine-stimulated time-response curve. The values of the area under the concentration-time curve for CPR (AUCCPR) between time 0 and 120 min were calculated using the trapezoidal rule and indicate the insulin-secreting response to arginine. The value of Arginine $\triangle \mathrm{CPR}$ was defined as the difference between maximal and basal levels of CPR during the arginine test.

\section{Lipid meal test}

Participants ingested a liquid meal $(750 \mathrm{kcal}, 500 \mathrm{~mL}$; Pulmocare, Abbott Japan, Tokyo, Japan) containing $53 \mathrm{~g}$ carbohydrate, $47 \mathrm{~g}$ lipid (including 20\% medium chain triglycerides), and $31 \mathrm{~g}$ protein, ${ }^{23}$ which has the highest carbohydrate and fat contents among the liquid test meals available at our hospital, after an overnight fast. Venous blood was obtained before and 30, 60, 120, and 180 min after ingestion, and plasma glucose and triglycerides were measured. Patients were instructed not to take sitagliptin or mitiglinide before the test. The AUCCPR values between time 0 and 180 min were calculated using the trapezoidal rule and indicate the insulinsecreting responses to the lipid meal test. The value of the lipid meal test $\triangle \mathrm{CPR}$ was defined as the difference between the maximal and basal levels of CPR during the lipid meal test.

\section{Treatment satisfaction}

Treatment satisfaction was a secondary outcome and was assessed using the Diabetes Treatment Satisfaction Questionnaire (DTSQ $)^{24-26}$ at baseline and at the end of the study. The overall treatment satisfaction score was calculated as the sum of DTSQ item 1, Satisfaction; item 4, Convenience; item 5, Flexibility; item 6, Understanding; item 7, Recommend to others; and item 8 , Wish to continue. Item 2, perceived hyperglycemia frequency, and item 3, perceived hypoglycemia frequency were treated as separate variables. The quality of life (QOL) instrument was not designed to measure treatment satisfaction related to the device.

\section{Statistical analysis}

Sample size was estimated to be 26 in each group to detect a 31.6 and $15.6 \mathrm{mg} / \mathrm{dL}$ decreased in FPG in the sitagliptin group ${ }^{27}$ and mitiglinide group, ${ }^{28}$ respectively, with an $\alpha$ of 0.05 (one tailed) and a $\beta$ of 0.20 with $80 \%$ 
power and a standardized effect size of 25. To take the dropout rate of $15 \%$ into account, the aim was to include 60 participants. Two analyses were conducted. Data that were missing for participants who discontinued the study were replaced with baseline data for the intention-to-treat analysis. In the second analysis, the only data included were from participants who completed the study (through the 16-week follow-up period). We performed a completed case analysis rather than an intention-to-treat analysis because there were few dropouts and their reasons for dropping out were unrelated to baseline values or their responses. Data are expressed as means \pm SD. The SPSS (V.22.0; SPSS, Chicago, Illinois, USA) was used for all the statistical analyses. Parameters were analyzed using the Wilcoxon signed-rank test for the intergroup comparison, and Mann-Whitney's U test for the internal group comparison. Associations between variables were assessed using Spearman's rank correlation coefficient. p Values $<0.05$ were considered as statistically significant.

\section{RESULTS}

\section{Baseline metabolic parameters}

Patients were recruited between May 2010 and July 2011, with the follow-up continuing for 16 weeks thereafter. Sixty patients consented to participate in the study and were screened and randomized (see online supplementary figure S1). The mean age of all participants was $63.5 \pm 12.0$ years, mean average diabetes duration was 9.7 \pm 9.2 years, mean average insulin dose was $21.3 \pm 8.8 \mathrm{U} /$ $24 \mathrm{~h}$, mean average duration of insulin use was 4.3 \pm 5.6 years, and mean body mass index (BMI) was 24.3 $\pm 4.2 \mathrm{~kg} / \mathrm{m}^{2}$ (see online supplementary table S1). FPG, HbAlc, and CPR immediately before the switch were $147.8 \pm 36.3 \mathrm{mg} / \mathrm{dL}, \quad 6.8 \pm 0.7 \%$, and $\quad 1.9 \pm 0.8 \mathrm{ng} / \mathrm{mL}$, respectively.

The groups were generally well balanced with respect to baseline demographics and disease characteristics, except for the serum creatinine level (sitagliptin creatinine (Cre) $0.69 \pm 0.19$, mitiglinide Cre $0.81 \pm 0.23$, $\mathrm{p}=0.036$ ) .

Of the 60 patients enrolled, 3 dropped out after the switch. The first dropout case was in the mitiglinide group (age, 70 years). The participant had returned to insulin therapy and developed a subconjunctival hemorrhage 8 weeks after the switch; the second case was in the sitagliptin group (age, 69 years). The participant voluntarily returned to insulin treatment and withdrew consent 3 days after the switch; and the third case (age, 57 years) returned to insulin therapy and became hyperglycemic 12 weeks after the switch (see online supplementary figure $\mathrm{S} 1$ ).

\section{Clinical outcomes}

FPG, which was the primary study outcome, increased significantly at the end of the study from $146.5 \pm 36.3$ to $168.0 \pm 38.8 \mathrm{mg} / \mathrm{dL}$ in the mitiglinide group, but did not change in the sitagliptin group.
Body weight, BMI, and waist circumference decreased significantly compared with baseline values in both groups at the end of the study (table 1). $\gamma$-GTP and triglycerides increased, and high-density lipoprotein cholesterol and adiponectin decreased, in the sitagliptin group, but not in the mitiglinide group. Sitagliptin increased the CPI, whereas mitiglinide significantly decreased the CPI.

The CPR responses after arginine were diminished in both groups. We investigated the liquid meal test results before and after switching to an OHA from insulin. The AUC CPR during the lipid meal test decreased significantly in the sitagliptin group when the participants switched from insulin.

Systolic blood pressure, blood urea nitrogen, AST, total cholesterol, small dense low-density lipoprotein, and tumor necrosis factor $\alpha$ had not changed significantly in either group at the end of the study.

\section{Changes in plasma fatty acid composition}

Sitagliptin significantly increased levels of lauric acid, myristic acid, $\gamma$-linolenic acid, $\alpha$-linolenic acid, eicosatrienoic acid, dihomo- $\gamma$-linolenic acid, and erucic acid, while mitiglinide had no effect (table 2). As a result, sitagliptin significantly decreased D5D, whereas it significantly increased D6D. Mitiglinide did not affect D5D or D6D. Levels of lauric acid, eicosatrienoic acid, and dihomo- $\gamma$-linolenic acid increased significantly in the sitagliptin group compared with the mitiglinide group (table 2).

\section{Treatment satisfaction}

The mean DTSQ scores for the sitagliptin and mitiglinide groups were $24.2 \pm 6.7$ and $22.3 \pm 1.6$, respectively, at baseline and $29.6 \pm 4.5$ and $28.4 \pm 1.3$ respectively, at the end of the study (table 3). No significant differences were observed in the change in treatment satisfaction scores between groups (sum of items 1, 4, 5, 6, 7, and 8) or for the changes in perceived frequency of hyperglycemia (item 2) or hypoglycemia (item 3) at the end point (table 3 ).

\section{Factors associated with improved $\mathrm{HbA1c}$}

We evaluated the correlation between factors and the change in $\mathrm{HbAlc}$ in a univariate analysis (see online supplementary table S2). Diabetes duration and use of insulin, physical balance, and fatty acid composition were not predictors of a change in HbAlc. Total insulin doses before switching from mealtime bolus insulin monotherapy to either sitagliptin or mitiglinide predicted changes in HbAlc in both groups. Patients whose mean total daily doses of rapid-acting insulin analog were 16.6 and 17.8 units were switched to sitagliptin and mitiglinide, respectively, without a change in the HbA1c level (see online supplementary figure S2). Total insulin doses/body weight predicted changes in HbA1c only in the sitagliptin group, but not in the mitiglinide group, whereas body weights did so only in the mitiglinide 
Table 1 Baseline patient characteristics and changes after 16 weeks

\begin{tabular}{|c|c|c|c|c|c|c|c|}
\hline & \multicolumn{2}{|l|}{ Sitagliptin } & \multirow[b]{2}{*}{ p Value* } & \multicolumn{2}{|l|}{ Mitiglinide } & \multirow[b]{2}{*}{ p Value* } & \multirow[b]{2}{*}{ p Valuet } \\
\hline & Before & After & & Before & After & & \\
\hline Body weight (kg) & $62.8 \pm 11.6$ & $61.5 \pm 11.5$ & 0.008 & $63.1 \pm 15.4$ & $61.7 \pm 14.9$ & 0.006 & 0.900 \\
\hline Body mass index $\left(\mathrm{kg} / \mathrm{m}^{2}\right)$ & $24.6 \pm 3.8$ & $24.1 \pm 3.6$ & 0.007 & $24.2 \pm 4.6$ & $23.7 \pm 4.5$ & 0.003 & 0.968 \\
\hline Waist circumference (cm) & $88.0 \pm 11.1$ & $86.1 \pm 10.0$ & 0.025 & $87.6 \pm 14.1$ & $85.8 \pm 12.9$ & 0.001 & 0.999 \\
\hline Systolic blood pressure (mm Hg) & $127.8 \pm 17.0$ & $131.7 \pm 14.4$ & 0.258 & $123.0 \pm 11.6$ & $127.2 \pm 16.6$ & 0.209 & 0.953 \\
\hline Fasting plasma glucose (mg/dL) & $145.2 \pm 28.2$ & $142.7 \pm 25.6$ & 0.616 & $146.5 \pm 36.3$ & $168.0 \pm 38.8$ & 0.032 & 0.033 \\
\hline $\mathrm{HbA} 1 \mathrm{c}(\%)$ & $6.6 \pm 0.5$ & $7.2 \pm 0.9$ & 0.000 & $7.0 \pm 0.8$ & $7.9 \pm 1.5$ & 0.000 & 0.153 \\
\hline BUN (mg/dL) & $15.6 \pm 4.5$ & $14.7 \pm 3.0$ & 0.250 & $16.8 \pm 5.2$ & $16.6 \pm 6.3$ & 0.821 & 0.579 \\
\hline Cre $(\mathrm{mg} / \mathrm{dL})$ & $0.69 \pm 0.19$ & $0.68 \pm 0.19$ & 0.742 & $0.81 \pm 0.24$ & $0.78 \pm 0.24$ & 0.030 & 0.166 \\
\hline $\begin{array}{l}\text { Serum aspartate } \\
\text { aminotransferase (IU/L) }\end{array}$ & $23.2 \pm 7.2$ & $24.8 \pm 9.5$ & 0.279 & $30.0 \pm 20.1$ & $27.3 \pm 17.0$ & 0.414 & 0.236 \\
\hline $\begin{array}{l}\text { Serum alanine aminotransferase } \\
\text { (IU/L) }\end{array}$ & $24.1 \pm 14.6$ & $27.8 \pm 9.5$ & 0.076 & $33.2 \pm 21.8$ & $31.8 \pm 21.1$ & 0.705 & 0.222 \\
\hline $\begin{array}{l}\text { Plasma } \gamma \text {-glutamyltransferase } \\
\text { (IU/L) }\end{array}$ & $32.2 \pm 17.0$ & $39.5 \pm 24.8$ & 0.013 & $31.9 \pm 20.9$ & $30.8 \pm 18.1$ & 0.752 & 0.060 \\
\hline Total cholesterol (mg/dL) & $182.5 \pm 30.1$ & $182.6 \pm 32.4$ & 0.979 & $181.6 \pm 23.8$ & $177.6 \pm 22.5$ & 0.341 & 0.485 \\
\hline Triglycerides (mg/dL) & $113.3 \pm 48.3$ & $168.4 \pm 46.2$ & 0.022 & $107.1 \pm 46.3$ & $116.1 \pm 49.6$ & 0.228 & 0.060 \\
\hline HDL-C (mg/dL) & $64.9 \pm 34.1$ & $52.5 \pm 13.9$ & 0.045 & $55.6 \pm 17.2$ & $54.6 \pm 15.5$ & 0.479 & 0.072 \\
\hline sdLDL (mg/dL) & $32.9 \pm 13.9$ & $36.8 \pm 17.0$ & 0.159 & $27.9 \pm 8.2$ & $28.2 \pm 10.0$ & 0.827 & 0.255 \\
\hline Fasting serum insulin (IU/L) & $10.0 \pm 6.6$ & $8.3 \pm 5.7$ & 0.517 & $8.9 \pm 8.0$ & $9.1 \pm 10.3$ & 0.879 & 0.118 \\
\hline CPR (ng/mL) & $1.8 \pm 0.7$ & $2.1 \pm 0.8$ & 0.079 & $2.0 \pm 0.8$ & $1.9 \pm 0.8$ & 0.529 & 0.638 \\
\hline Arginine $\triangle \mathrm{CPR}(\mathrm{ng} / \mathrm{mL})$ & $2.8 \pm 1.4$ & $2.3 \pm 1.5$ & 0.049 & $2.7 \pm 1.6$ & $2.4 \pm 1.8$ & 0.028 & 0.203 \\
\hline Arginine AUC CPR $\times 10^{2}$ & $4.6 \pm 1.6$ & $4.4 \pm 2.0$ & 0.587 & $4.7 \pm 2.1$ & $4.6 \pm 2.1$ & 0.778 & 0.731 \\
\hline Lipid meal test $\Delta$ CPR $(\mathrm{ng} / \mathrm{mL})$ & $4.9 \pm 2.4$ & $4.3 \pm 1.3$ & 0.109 & $5.1 \pm 2.6$ & $4.6 \pm 2.8$ & 0.166 & 0.954 \\
\hline Lipid meal test AUC CPR $\times 10^{2}$ & $8.7 \pm 3.6$ & $8.1 \pm 3.1$ & 0.038 & $9.2 \pm 3.6$ & $8.5 \pm 0.4$ & 0.216 & 0.870 \\
\hline 1,5-anhydroglucitol (mg/mL) & $12.6 \pm 6.4$ & $9.5 \pm 5.3$ & 0.001 & $11.7 \pm 6.7$ & $8.2 \pm 7.3$ & 0.000 & 0.736 \\
\hline Leptin (ng/mL) & $8.7 \pm 6.5$ & $8.5 \pm 7.1$ & 0.855 & $10.5 \pm 13.4$ & $8.9 \pm 11.0$ & 0.082 & 0.215 \\
\hline Adiponectin (mg/mL) & $4.5 \pm 3.9$ & $4.0 \pm 3.4$ & 0.019 & $5.0 \pm 4.4$ & $4.4 \pm 3.1$ & 0.138 & 0.904 \\
\hline TNF- $\alpha(p g / m L)$ & $2.9 \pm 1.7$ & $1.6 \pm 1.7$ & 0.267 & $2.1 \pm 1.9$ & $2.0 \pm 1.6$ & 0.801 & 0.325 \\
\hline HOMA-b & $44.6 \pm 28.9$ & $38.0 \pm 25.0$ & 0.226 & $49.0 \pm 23.5$ & $44.5 \pm 28.4$ & 0.351 & 0.773 \\
\hline $\mathrm{CPI}$ & $1.3 \pm 0.5$ & $1.4 \pm 0.6$ & 0.052 & $1.5 \pm 0.7$ & $1.2 \pm 0.7$ & 0.021 & 0.003 \\
\hline
\end{tabular}

Data are means \pm SD.

${ }^{*} p$ Value for the intragroup comparison (baseline vs 16 weeks).

tp Value for the intergroup comparison (change from baseline between groups).

AUC, area under the curve; BUN, blood urea nitrogen; CPI, C peptide immunoreactivity index; CPR, C peptide immunoreactivity; Cre, creatinine; HbA1c, glycated hemoglobin; HDL-C, high-density lipoprotein cholesterol; HOMA, homeostasis model assessment of insulin resistance; sdLDL, small dense low-density lipoprotein; TNF, tumor necrosis factor.

group, but not in the sitagliptin group (see online supplementary table S2). Use of $>0.27 \mathrm{IU} / \mathrm{kg}$ of a rapid-acting insulin analog predicted an increase in HbA1c after switching to sitagliptin (see online supplementary figure S2).

The CPI was also a predictor for a change in HbAlc in the sitagliptin group, but not in the mitiglinide group; patients with a CPI $<1.4$ developed a worse HbA1c after switching to sitagliptin (see online supplementary figure S3). Docosahexaenoic acid (DHA) and eicosapentaenoic acid (EPA) levels did not predict a sitagliptin-mediated improvement in glycemic control. Changes in HbA1c in the mitiglinide group were negatively associated with changes in body weight and BMI.

\section{DISCUSSION}

This is the first report showing the feasibility of substituting an OHA for insulin injection therapy in an open- label randomized, parallel-group study. Our results show that FPG increased significantly in participants receiving mitiglinide, whereas no changes were observed in those administered sitagliptin. Mealtime bolus insulin monotherapy was superior to sitagliptin and mitiglinide for controlling HbA1c. Sitagliptin acted on FPG, whereas mitiglinide may act on the postprandial plasma glucose level to achieve a similar HbAlc after the switch from a bolus insulin regimen.

The majority of patients with type 2 diabetes treated with insulin have difficulty achieving or maintaining target glycemic control without an associated weight gain. ${ }^{29}$ In this study, body weight, BMI, and waist circumference decreased significantly in both OHA groups after the switch from insulin. OHAs are more effective than bolus insulin monotherapy for maintaining weight in patients with type 2 diabetes at high risk for cardiovascular disease. We did not show a positive effect of OHAs on lipid profiles or blood pressure control. This could 
Table 2 Changes in plasma fatty acid composition

\begin{tabular}{|c|c|c|c|c|c|c|c|}
\hline & \multicolumn{2}{|l|}{ Sitagliptin } & \multirow{2}{*}{$\begin{array}{l}\text { p } \\
\text { Value* }^{*}\end{array}$} & \multicolumn{2}{|l|}{ Mitiglinide } & \multirow{2}{*}{$\begin{array}{l}\text { p } \\
\text { Value* }^{*}\end{array}$} & \multirow{2}{*}{$\begin{array}{l}\mathbf{p} \\
\text { Valuet }\end{array}$} \\
\hline & Before & After & & Before & After & & \\
\hline C12:0 (lauric acid) & $1.6 \pm 1.0$ & $2.7 \pm 2.2$ & 0.011 & $1.7 \pm 1.2$ & $1.7 \pm 0.7$ & 0.988 & 0.024 \\
\hline C14:0 (myristic acid) & $23.7 \pm 9.8$ & $32.3 \pm 17.5$ & 0.015 & $24.9 \pm 12.5$ & $26.3 \pm 9.2$ & 0.586 & 0.083 \\
\hline C16:0 (palmitic acid) & $707.9 \pm 182.1$ & $779.3 \pm 322.1$ & 0.212 & $707.4 \pm 129.9$ & $680.0 \pm 125.6$ & 0.270 & 0.114 \\
\hline $\begin{array}{l}\text { C16:1n-7 (palmitoleic } \\
\text { acid) }\end{array}$ & $71.9 \pm 27.5$ & $78.4 \pm 37.1$ & 0.316 & $75.8 \pm 37.8$ & $72.6 \pm 40.1$ & 0.521 & 0.233 \\
\hline C18:0 (stearic acid) & $213.0 \pm 42.9$ & $232.8 \pm 86.2$ & 0.244 & $204.0 \pm 30.3$ & $197.9 \pm 31.9$ & 0.269 & 0.148 \\
\hline C18:1n-9 (oleic acid) & $644.2 \pm 245.2$ & $747.8 \pm 405.7$ & 0.151 & $606.9 \pm 125.6$ & $607.0 \pm 142.6$ & 0.997 & 0.165 \\
\hline C18:2n-6 (linoleic acid) & $818.7 \pm 178.1$ & $845.0 \pm 279.2$ & 0.514 & $781.0 \pm 151.6$ & $772.4 \pm 165.3$ & 0.746 & 0.468 \\
\hline $\begin{array}{l}\text { C18:3n-6 ( } \gamma \text {-linolenic } \\
\text { acid) }\end{array}$ & $10.0 \pm 4.7$ & $11.6 \pm 6.5$ & 0.022 & $9.1 \pm 4.5$ & $9.3 \pm 3.8$ & 0.805 & 0.159 \\
\hline $\begin{array}{l}\text { C18:3n-3 ( } \alpha \text {-linolenic } \\
\text { acid) }\end{array}$ & $26.1 \pm 11.3$ & $35.8 \pm 26.7$ & 0.029 & $23.8 \pm 8.6$ & $25.1 \pm 10.9$ & 0.487 & 0.073 \\
\hline C20:0n-6 (arachidic acid) & $7.1 \pm 1.4$ & $7.5 \pm 2.4$ & 0.337 & $7.2 \pm 1.1$ & $6.9 \pm 1.1$ & 0.083 & 0.145 \\
\hline $\begin{array}{l}\text { C20:1n9 (eicosenoic } \\
\text { acid) }\end{array}$ & $5.8 \pm 2.5$ & $6.8 \pm 4.7$ & 0.290 & $5.6 \pm 2.8$ & $5.1 \pm 1.1$ & 0.329 & 0.163 \\
\hline $\begin{array}{l}\text { C20:2n6 (eicosadienoic } \\
\text { acid) }\end{array}$ & $5.9 \pm 1.7$ & $6.3 \pm 2.2$ & 0.332 & $5.7 \pm 1.3$ & $5.6 \pm 1.3$ & 0.637 & 0.280 \\
\hline $\begin{array}{l}\text { C20:3n9 (eicosatrienoic } \\
\text { acid) }\end{array}$ & $2.2 \pm 1.2$ & $2.6 \pm 1.4$ & 0.012 & $2.0 \pm 1.1$ & $1.8 \pm 1.0$ & 0.436 & 0.027 \\
\hline $\begin{array}{l}\text { C20:3n-6 } \\
\text { (dihomo- } \gamma \text {-linolenic acid) }\end{array}$ & $35.8 \pm 11.3$ & $39.6 \pm 14.6$ & 0.030 & $37.7 \pm 12.2$ & $35.7 \pm 10.2$ & 0.248 & 0.018 \\
\hline $\begin{array}{l}\text { C20:4n-6 (arachidonic } \\
\text { acid) }\end{array}$ & $189.0 \pm 41.0$ & $177.6 \pm 53.8$ & 0.065 & $178.7 \pm 32.1$ & $163.7 \pm 23.2$ & 0.016 & 0.663 \\
\hline $\begin{array}{l}\text { C20:5n-3 } \\
\text { (eicosapentaenoic acid) }\end{array}$ & $73.0 \pm 30.2$ & $70.1 \pm 32.2$ & 0.579 & $78.8 \pm 39.9$ & $73.2 \pm 42.7$ & 0.511 & 0.783 \\
\hline C22:0 (behenic acid) & $18.3 \pm 3.7$ & $17.9 \pm 5.4$ & 0.583 & $18.4 \pm 4.0$ & $17.8 \pm 4.7$ & 0.269 & 0.836 \\
\hline C22:1n-9 (erucic acid) & $1.5 \pm 0.5$ & $1.8 \pm 0.9$ & 0.047 & $1.6 \pm 0.6$ & $1.8 \pm 0.5$ & 0.121 & 0.674 \\
\hline $\begin{array}{l}\text { C22:4n-6 } \\
\text { (docosatetraenoic acid) }\end{array}$ & $5.0 \pm 1.6$ & $5.4 \pm 2.3$ & 0.182 & $4.7 \pm 1.4$ & $4.6 \pm 1.5$ & 0.588 & 0.154 \\
\hline $\begin{array}{l}\text { C22:5n-3 } \\
\text { (docosapentaenoic acid) }\end{array}$ & $21.1 \pm 5.7$ & $23.1 \pm 9.2$ & 0.148 & $21.3 \pm 6.9$ & $21.5 \pm 9.3$ & 0.921 & 0.352 \\
\hline $\begin{array}{l}\text { C22:6n-3 } \\
\text { (docosahexaenoic acid) }\end{array}$ & $156.0 \pm 33.1$ & $149.8 \pm 37.5$ & 0.357 & $163.8 \pm 50.1$ & $149.2 \pm 39.7$ & 0.057 & 0.404 \\
\hline C24:1 (nervonic acid) & $34.7 \pm 6.2$ & $32.0 \pm 6.4$ & 0.007 & $37.9 \pm 7.4$ & $36.8 \pm 7.7$ & 0.297 & 0.250 \\
\hline SCD-16 (C16:1/C16:0) & $0.10 \pm 0.03$ & $0.10 \pm 0.02$ & 0.755 & $0.10 \pm 0.04$ & $0.10 \pm 0.04$ & 0.971 & 0.840 \\
\hline SCD-18 (C18:1n-9/C18:0) & $2.97 \pm 0.61$ & $3.12 \pm 0.77$ & 0.195 & $2.97 \pm 0.44$ & $3.07 \pm 0.53$ & 0.151 & 0.685 \\
\hline D6D (C18:3n-6/C18:2n6) & $0.012 \pm 0.005$ & $0.013 \pm 0.007$ & 0.023 & $0.012 \pm 0.007$ & $0.012 \pm 0.005$ & 0.917 & 0.365 \\
\hline D5D (C20:4n-6/C20:3n6) & $5.70 \pm 1.88$ & $4.75 \pm 1.31$ & 0.001 & $5.30 \pm 2.24$ & $4.90 \pm 1.37$ & 0.253 & 0.199 \\
\hline
\end{tabular}

Data are means \pm SD.

${ }^{*} p$ Value for the intragroup comparison (baseline vs 16 weeks).

tp Value for the intergroup comparison (change from baseline between groups).

D5D, $\Delta 5$ desaturase.

be related to the limitation that the observation period was too short to detect beneficial effects on lipid profiles or blood pressure. Sitagliptin-mediated and mitiglinidemediated improvements in glycemic control were independent of the lipid profile or insulin secretion (see online supplementary table S2), suggesting that unique and as yet unrecognized mechanisms may underlie the actions. Indeed, insulin secretion during the arginine challenge and lipid meal tests decreased significantly in the sitagliptin group after the switch from insulin. The effects of glucagon-like peptide-1 on glucagon secretion, gastric emptying, and the autonomic nervous system may explain such an effect of sitagliptin. ${ }^{30}$ In addition, our results suggest that glinides also have a glucose-lowering effect independent of their effect on insulin secretion.

In contrast to a previous report, ${ }^{31}$ DHA and EPA levels did not predict the sitagliptin-mediated improvement in glycemic control (see online supplementary table S2). In addition, this is the first study to demonstrate sitagliptin-mediated and mitiglinide-mediated changes in serum fatty acid profiles in humans or animals. Sitagliptin, but not mitiglinide, dynamically altered fatty acid composition; it increased serum levels of fatty acids, such as lauric acid, myristic acid, $\gamma$-linolenic acid, $\alpha$-linolenic acid, eicosatrienoic acid, dihomo- $\gamma$-linolenic acid, and erucic acid. The effects of sitagliptin on fatty 
Table 3 Changes in treatment satisfaction

\begin{tabular}{|c|c|c|c|c|c|c|c|}
\hline & \multicolumn{2}{|c|}{ Sitagliptin } & \multirow[b]{2}{*}{ p Value* } & \multicolumn{2}{|c|}{ Mitiglinide } & \multirow[b]{2}{*}{ p Value* } & \multirow[b]{2}{*}{ p Valuet } \\
\hline & Before & After & & Before & After & & \\
\hline Q1 & $4.1 \pm 1.7$ & $4.9 \pm 1.1$ & 0.057 & $4.3 \pm 0.3$ & $4.8 \pm 0.3$ & 0.197 & 0.620 \\
\hline Q2 & $3.1 \pm 1.4$ & $2.6 \pm 1.7$ & 0.264 & $2.6 \pm 0.3$ & $2.9 \pm 0.4$ & 0.412 & 0.170 \\
\hline Q3 & $2.3 \pm 1.9$ & $1.9 \pm 1.9$ & 0.358 & $1.3 \pm 0.2$ & $1.9 \pm 0.4$ & 0.166 & 0.110 \\
\hline Q4 & $3.7 \pm 1.7$ & $5.2 \pm 0.9$ & 0.000 & $3.0 \pm 0.4$ & $5.0 \pm 0.3$ & 0.000 & 0.268 \\
\hline Q5 & $3.7 \pm 1.3$ & $4.7 \pm 1.3$ & 0.006 & $3.1 \pm 0.4$ & $4.8 \pm 0.3$ & 0.000 & 0.146 \\
\hline Q6 & $4.1 \pm 1.3$ & $4.6 \pm 1.0$ & 0.043 & $4.6 \pm 0.2$ & $4.5 \pm 0.3$ & 0.887 & 0.154 \\
\hline Q7 & $4.3 \pm 1.5$ & $5.0 \pm 0.8$ & 0.013 & $3.7 \pm 0.4$ & $4.4 \pm 0.3$ & 0.052 & 0.931 \\
\hline Q8 & $4.1 \pm 1.6$ & $5.1 \pm 1.0$ & 0.002 & $3.7 \pm 0.4$ & $4.8 \pm 0.3$ & 0.008 & 1.000 \\
\hline Sum & $24.2 \pm 6.7$ & $29.6 \pm 4.5$ & 0.000 & $22.3 \pm 1.6$ & $28.4 \pm 1.3$ & 0.001 & 0.759 \\
\hline
\end{tabular}

acid profiles were independent of its effects on glycemic control, insulin sensitivity, and cardiovascular markers (data not shown). D5D and D6D catalyze the synthesis of long-chain n-6 and n-3 polyunsaturated fatty acids (PUFAs), and their activities can be estimated using PUFA product-to-precursor ratios. ${ }^{32} 33$ The D5D activity index is negatively associated with insulin resistance, ${ }^{33} 34$ onset of newly diagnosed type 2 diabetes, ${ }^{32}$ and an adverse profile of several metabolic risk factors in patients with metabolic syndrome ${ }^{35}$ in cross-sectional studies. Insulin activates D5D in patients with diabetes. ${ }^{36}$ However, another cross-sectional study showed that patients with poorly controlled type 2 diabetes have a higher D5D activity index, ${ }^{37}$ and improving glycemic control with intensive insulin therapy significantly decreases D5D in patients with type 2 diabetes. ${ }^{38}$ Our study is the first to show the effect of a dipeptidyl peptidase-4 (DPP-4) inhibitor on D5D and D6D. After the switch from insulin, sitagliptin significantly decreased D5D, whereas it significantly increased D6D. Neither the baseline levels of D5D nor D6D predicted the sitagliptin-mediated improvement in glycemic control. Thus, sitagliptin, but not mitiglinide, may exert unique pleiotropic effects on fatty acid composition. The molecular mechanisms underlying the sitagliptinmediated effects on fatty acid metabolism should be pursued in future studies.

Finally, patients who took sitagliptin or mitiglinide after switching from insulin ameliorated overall QOL as assessed by the DTSQ. The DTSQ was developed to enable respondents to evaluate their current treatment in relation to their previous treatment. Among the DTSQ subscale scores, convenience of treatment, flexibility of treatment, and satisfaction to continue current treatment showed a significant increase after switching from insulin in both groups. Previously, it was reported that less treatment satisfaction is related to insulin treatment. ${ }^{39}$ In addition, the satisfaction with treatment is reported to significantly correlate with adherence. ${ }^{40}$ Therefore, in this study, convenience of OHAs may contribute to satisfaction with treatment.
Patient factors associated with changes in $\mathrm{HbAlc}$ remain unclear after switching from a bolus insulin regimen to an OHA. Total insulin doses before switching from mealtime bolus insulin monotherapy to either sitagliptin or mitiglinide predicted changes in $\mathrm{HbAlc}$ in both groups. Interestingly, total insulin doses/body weight predicted changes in HbAlc only in the sitagliptin group, but not in the mitiglinide group, whereas body weights did so only in the mitiglinide group, but not in the sitagliptin group. This may be because the effect of mitiglinide, but not sitagliptin, is dependent on plasma drug concentration that is decreased in increased body weight.

The baseline CPI significantly predicted changes in HbAlc only in the sitagliptin group. One study reported that the CPI is associated with pancreatic $\beta$-cell function in Japanese patients with type 2 diabetes; patients with CPI $<0.8$ usually require insulin therapy. ${ }^{41}$ Our study is the first to prospectively demonstrate usefulness of the CPI for "tailor-made" diabetic medicine. Patients with a $\mathrm{CPI}<1.4$ had worse HbAlc levels after switching to sitagliptin.

Our study has some limitations. First, mealtime dosing of rapid-acting insulin analog monotherapy may be a less common regimen in the diabetes treatment strategy. However, we previously showed that approximately one-half of the Japanese patients with type 2 diabetes retain $\beta$-cell function enough to achieve appropriate control of FPG by rapid-acting insulin analog monotherapy. ${ }^{1}$ We hypothesized that such patients may be considered switch to DPP-4 inhibitors or glinides. Second, it was unexpected that all the study participants experienced exacerbation in glycemic control after switching from bolus insulin regimen to OHAs. However, as a result, the potent effect of mealtime dosing of rapid-acting insulin analog monotherapy on glycemic control was confirmed in patients with type 2 diabetes. As we showed in this study, insulin doses and $\beta$-cell function may predict feasibility to sitagliptin therapy. Third, we intended to compare the efficacy of sitagliptin versus mitiglinide in controlling FPG after switching from 
insulin therapy. Therefore, we designed a parallel group study just comparing these agents. However, setting the control group that continued the insulin therapy further enables one to compare the efficacy of bolus insulin and OHAs in controlling FPG and HbA1c.

In conclusion, nadir FPG and HbAlc were exacerbated in patients receiving sitagliptin or mitiglinide after switching from mealtime dosing of a rapid-acting insulin analog. Mean FPG increased significantly in the mitiglinide group, whereas it remained unchanged in the sitagliptin group, but participants achieved similar glycemic control after switching from the bolus insulin regimen. In contrast, switching to sitagliptin or mitiglinide decreased BMI and waist circumference and increased QOL. Sitagliptin, but not mitiglinide, may exert unique pleiotropic effects on fatty acid composition. Patients whose mean total daily doses of rapid-acting insulin analog were 16.6 and 17.8 units were switched to sitagliptin and mitiglinide, respectively, without a change in the HbA1c level. Additional therapy to sitagliptin or mitiglinide is clearly required to obtain equivalent glycemic control in patients using higher dosing of insulin.

Contributors TT is the guarantor. YT designed the study, recruited the patients, analyzed the data, and wrote the manuscript. YK analyzed and interpreted the data. AT, K-iK, and YK collected clinical information. SK initiated and organized the study. All the authors have read and approved the final manuscript.

Funding This work was supported by Grants-in-Aid from the Ministry of Education, Culture, Sports, Science, and Technology, Japan and research grants from Novo Nordisk, Eli Lilly, Ono, MSD, Kissei, and Takeda pharmaceutical companies (to TT and SK).

Competing interests None declared.

Patient consent Obtained.

Ethics approval UMIN 000007051.

Provenance and peer review Not commissioned; externally peer reviewed.

Data sharing statement No additional data are available.

Open Access This is an Open Access article distributed in accordance with the Creative Commons Attribution Non Commercial (CC BY-NC 4.0) license, which permits others to distribute, remix, adapt, build upon this work noncommercially, and license their derivative works on different terms, provided the original work is properly cited and the use is non-commercial. See: http:// creativecommons.org/licenses/by-nc/4.0/

\section{REFERENCES}

1. Takamura $\mathrm{T}$, Sakurai M, Nakamura M, et al. Factors associated with improvement of fasting plasma glucose level by mealtime dosing of a rapid-acting insulin analog in type 2 diabetes. Diabetes Res Clin Pract 2007;75:278-84.

2. Anderson JH Jr., Brunelle RL, Koivisto VA, et al. Improved mealtime treatment of diabetes mellitus using an insulin analogue. Clin Ther 1997; 19:62-72.

3. Del Prato S. In search of normoglycaemia in diabetes: controlling postprandial glucose. Int J Obes Relat Metab Disord 2002;26: S9-S17.

4. Mukhopadhyay A, Oh SW, Tissenbaum HA. Worming pathways to and from DAF-16/FOXO. Exp Gerontol 2006;41:928-34.

5. Carstensen B, Witte DR, Friis S. Cancer occurrence in Danish diabetic patients: duration and insulin effects. Diabetologia 2012;55:948-58.

6. Komura T, Mizukoshi E, Kita $\mathrm{Y}$, et al. Impact of diabetes on recurrence of hepatocellular carcinoma after surgical treatment in patients with viral hepatitis. Am J Gastroenterol 2007;102:1939-46.
7. Kita Y, Mizukoshi E, Takamura T, et al. Impact of diabetes mellitus on prognosis of patients infected with hepatitis $\mathrm{C}$ virus. Metabolism 2007;56:1682-8.

8. Kalmijn S, Feskens EJ, Launer LJ, et al. Glucose intolerance, hyperinsulinaemia and cognitive function in a general population of elderly men. Diabetologia 1995;38:1096-102.

9. Luchsinger JA, Tang MX, Shea S, et al. Hyperinsulinemia and risk of Alzheimer disease. Neurology 2004;63:1187-92.

10. Kuusisto J, Koivisto K, Mykkänen L, et al. Association between features of the insulin resistance syndrome and Alzheimer's disease independently of apolipoprotein E4 phenotype: cross sectional population based study. BMJ 1997;315:1045-9.

11. Ott A, Stolk RP, van Harskamp F, et al. Diabetes mellitus and the risk of dementia: the Rotterdam Study. Neurology 1999;53:1937-42.

12. Curb JD, Rodriguez BL, Abbott RD, et al. Longitudinal association of vascular and Alzheimer's dementias, diabetes, and glucose tolerance. Neurology 1999;52:971-5.

13. Luchsinger JA, Tang MX, Stern $\mathrm{Y}$, et al. Diabetes mellitus and risk of Alzheimer's disease and dementia with stroke in a multiethnic cohort. Am J Epidemiol 2001;154:635-41.

14. Cushman WC, Evans GW, Byington RP, et al., ACCORD Study Group. Effects of intensive blood-pressure control in type 2 diabetes mellitus. N Engl J Med 2010;362:1575-85.

15. Chyan YJ, Chuang LM. Dipeptidyl peptidase-IV inhibitors: an evolving treatment for type 2 diabetes from the incretin concept. Recent patents on endocrine. Metab Immune Drug Discov 2007;1:15-24

16. Amori RE, Lau J, Pittas AG. Efficacy and safety of incretin therapy in type 2 diabetes: systematic review and meta-analysis. JAMA 2007;298:194-206.

17. Nauck MA, Meininger G, Sheng D, et al. Efficacy and safety of the dipeptidyl peptidase-4 inhibitor, sitagliptin, compared with the sulfonylurea, glipizide, in patients with type 2 diabetes inadequately controlled on metformin alone: a randomized, double-blind, non-inferiority trial. Diabetes Obes Metab 2007;9:194-205.

18. Ohnota $\mathrm{H}$, Koizumi $\mathrm{T}$, Tsutsumi $\mathrm{N}$, et al. Novel rapid- and short-acting hypoglycemic agent, a calcium(2s)-2-benzyl-3(cis-hexahydro-2-isoindolinylcarbonyl) propionate (KAD-1229) that acts on the sulfonylurea receptor: comparison of effects between KAD-1229 and gliclazide. J Pharmacol Exp Ther 1994;269:489-95.

19. Marre M, Shaw J, Brändle $M$, et al. Liraglutide, a once-daily human GLP-1 analogue, added to a sulphonylurea over 26 weeks produces greater improvements in glycaemic and weight control compared with adding rosiglitazone or placebo in subjects with type 2 diabetes (LEAD-1 SU). Diabet Med 2009;26:268-78.

20. Neutel JM, Zhao C, Karyekar CS. Adding saxagliptin to metformin extended release (XR) or uptitration of metformin XR: efficacy on daily glucose measures. Diabetes Ther 2013;4:269-83.

21. Katz A, Nambi SS, Mather K, et al. Quantitative insulin sensitivity check index: a simple, accurate method for assessing insulin sensitivity in humans. J Clin Endocrinol Metab 2000;85:2402-10.

22. Tsuchiyama N, Takamura $\mathrm{T}$, Ando $\mathrm{H}$, et al. Possible role of alpha-cell insulin resistance in exaggerated glucagon responses to arginine in type 2 diabetes. Diabetes Care 2007;30:2583-7.

23. Angelillo VA, Bedi S, Durfee D, et al. Effects of low and high carbohydrate feedings in ambulatory patients with chronic obstructive pulmonary disease and chronic hypercapnia. Annal Int Med 1985;103:883-5.

24. Bradley C. Diabetes treatment satisfaction questionnaire. Change version for use alongside status version provides appropriate solution where ceiling effects occur. Diabetes Care 1999;22:530-2.

25. Bradley C, Gamsu DS. Guidelines for encouraging psychological well-being: report of a Working Group of the World Health Organization Regional Office for Europe and International Diabetes Federation European Region St Vincent Declaration Action Programme for Diabetes. Diabet Med 1994;11:510-16.

26. Ishii H, Bradley C, Riazi A, et al. The Japanese version of the Diabetes Treatment Satisfaction Questionnaire (DTSQ): translation and clinical evaluation. Igaku No Ayumi 2000;192:809-14.

27. Mohan V, Yang W, Son HY, et al. Efficacy and safety of sitagliptin in the treatment of patients with type 2 diabetes in China, India, and Korea. Diabetes Res Clin Pract 2009;83:106-16.

28. Kaku K, Tanaka S, Origasa $\mathrm{H}$, et al. Effect of mitiglinide on glycemic control over 52 weeks in Japanese type 2 diabetic patients insufficiently controlled with pioglitazone monotherapy. Endocr $J$ 2009:56:739-46.

29. Calvert MJ, McManus RJ, Freemantle N. Management of type 2 diabetes with multiple oral hypoglycaemic agents or insulin in primary care: retrospective cohort study. $\mathrm{Br} J$ Gen Pract 2007; 57:455-60. 
30. Eliasson B, Möller-Goede D, Eeg-Olofsson K, et al. Lowering of postprandial lipids in individuals with type 2 diabetes treated with alogliptin and/or pioglitazone: a randomised double-blind placebo-controlled study. Diabetologia 2012;55:915-25.

31. Senmaru T, Fukui M, Kobayashi K, et al. DPP-IV inhibitors is effective in patients with type 2 diabetes with high serum eicosapentaenoic acid concentrations. J Diabetes Invest 2012;3:498-502.

32. Kroger J, Schulze MB. Recent insights into the relation of $\Delta 5$ desaturase and $\Delta 6$ desaturase activity to the development of type 2 diabetes. Curr Opin Lipidol 2012;23:4-10.

33. Vessby B, Gustafsson IB, Tengblad S, et al. Desaturation and elongation of fatty acids and insulin action. Ann N Y Acad Sci 2002;967:183-95.

34. Warensjo E, Rosell M, Hellenius ML, et al. Associations between estimated fatty acid desaturase activities in serum lipids and adipose tissue in humans: links to obesity and insulin resistance. Lipids Health Dis 2009;8:37.

35. Mayneris-Perxachs J, Guerendiain M, Castellote Al, et al. Plasma fatty acid composition, estimated desaturase activities, and their relation with the metabolic syndrome in a population at high risk of cardiovascular disease. Clin Nutr 2014;33:90-7.
36. Boustani S, Causse JE, Descomps B, et al. Direct in vivo characterization of delta 5 desaturase activity in humans by deuterium labeling: effect of insulin. Metabolism 1989;38:315-21.

37. Imamura S, Morioka T, Yamazaki Y, et al. Plasma polyunsaturated fatty acid profile and delta- 5 desaturase activity are altered in patients with type 2 diabetes. Metabolism 2014;63:1432-8.

38. Sartore G, Lapolla A, Reitano R, et al. Desaturase activities and metabolic control in type 2 diabetes. Prostaglandins Leukot Essent Fatty Acids 2008;79:55-8.

39. Nicolucci A, Giorgino R, Cucinotta $D$, et al. Validation of the Italian version of the WHO-Well-Being Questionnaire (WHO-WBQ) and the WHO-Diabetes Treatment Satisfaction Questionnaire (WHO-DTSQ). Diabetes Nutr Metab 2004;17:235-43.

40. Jordan J, Cahn P, Goebel F, et al. Abacavir compared to protease inhibitors as part of HAART regimens for treatment of HIV infection: patient satisfaction and implications for adherence. AIDS Patient Care STDS 2005;19:9-18.

41. Funakoshi S, Fujimoto S, Hamasaki A, et al. Analysis of factors influencing pancreatic beta cell function in Japanese patients with type 2 diabetes: association with body mass index and duration of diabetic exposure. Diabetes Res Clin Pract 2008;82: 353-8. 